\title{
Formação das Mulheres nas Escolas de Medicina
}

\author{
Women and Medical Schools
}

Rebeca Contrera Ávila

\section{PALAVRAS-CHAVE}

- Educação Médica.

- Mulheres.

- Preconceito de Gênero.

- Identidade de Gênero.

\section{KEYWORDS}

- Medical Education.

- Women.

- Prejudice Based on Gender.

- Gender Identity.

Recebido em: 09/03/2012

Reeencaminhado em: 22/09/2012

Reeencaminhado em: 10/02/2013

Aprovado em: 07/04/2014

\section{RESUMO}

A feminização da medicina tem sido apontada por diversas pesquisas como uma das mudanças de maior impacto sobre a profissão médica. Embora na última década tenham representado a maior parte dos estudantes nas escolas de Medicina e a maior parte dos novos profissionais registrados, as mulheres continuam a sofrer significativo impacto negativo provocado por estereótipos sexistas e discriminação de gênero. Este artigo contempla uma revisão de literatura sobre as questões contemporâneas que envolvem a educação das mulheres nas escolas de Medicina. O objetivo deste artigo é apresentar algumas das principais questões abordadas pela comunidade acadêmica com respeito a essa problemática e dar visibilidade às estratégias adotadas por algumas instituições de ensino como tentativa de promover a equidade de gênero nas escolas de formação médica.

\section{ABSTRACT}

The feminization of medicine has been pointed out by several researchers as one of the most influential changes in the profession. Despite the fact that the majority of medical students and registered professionals in the past decade were female, women still experience a significant negative impact, caused by sexist stereotypes and gender discrimination. This article aims to review literature on the contemporary issues involving women's education in medical schools. The objective is to present some of the main points discussed by the academic community with regard to this issue and to publicize several educational institutions' strategies in an attempt to promote gender equality in medical schools. 


\section{INTRODUÇÃO}

"Novos dados confirmam predominância das mulheres na graduação médica"1 (p.5)

"Mulheres médicas são maioria entre os mais jovens"2(p.8)

Essas manchetes foram veiculadas pelo departamento de imprensa do Conselho Regional de Medicina do Estado de São Paulo (Cremesp) para destacar uma mudança histórica sem precedentes no Estado que conta com o maior número de médicos no Brasil: as mulheres representam a maioria dos novos médicos registrados ${ }^{3}$.

Seguindo uma tendência mundial, até a década de 1960 a medicina no Brasil era exercida majoritariamente por homens ${ }^{4,5}$. Foi somente a partir da década de 1970 que as faculdades de Medicina passaram a ser um espaço de maior circulação das mulheres, aumentando gradativamente ao longo das décadas de 1980 e 1990 e mais aceleradamente na primeira década do século $\mathrm{XXI}^{-9}$. Nas últimas décadas, tem sido rompido o hiato de gênero que durante séculos cerceou a entrada das mulheres na profissão médica. De uma profissão dominada pelos homens, a medicina passa a ser uma profissão em que a maioria dos novos licenciados são mulheres, e, ao menos nos países mais urbanizados e industrializados, essa tendência representa um fenômeno global ${ }^{2,3,8,10,11}$. Em países como Reino Unido, Austrália, Canadá, Estados Unidos ${ }^{12,13}$, Suíça, Alemanha ${ }^{14,15} \mathrm{e} \mathrm{Holanda}^{16}$, as mulheres representam em torno de $60 \%$ dos novos licenciados. Na Austrália e em Portugal, no final da década de 1990, elas já representavam, respectivamente, $68 \%$ e $69 \%$ dos novos ingressantes ${ }^{13,10}$.

A feminização da medicina tem desencadeado grandes mudanças nessa categoria profissional, e uma das mudanças de maior impacto tem sido a escassez de mão de obra em algumas especialidades. A "preferência" das mulheres por especialidades como Pediatria e Ginecologia e Obstetrícia tem ocasionado um déficit de profissionais, por exemplo, nas especialidades cirúrgicas ${ }^{11,12,14,17,18}$. No Japão, pesquisas realizadas pelo Departamento de Medicina e Saúde Coletiva da Escola de Medicina da Universidade de Yamaguchi alertam para o fato de que o país passa por uma crise na medicina por falta de profissionais em determinadas especialidades e que essa carência será agravada pelo aumento das mulheres na medicina ${ }^{17}$. Nos Estados Unidos, governo e corporações médicas se preocupam com o fato de que, em um futuro próximo, haverá um déficit de médicos(as) cardiovasculares no país ${ }^{11,18}$. Anthony De Maria, editor-chefe do Journal of the American College of Cardiology, explica que o aumento do número de mulheres médicas tem gerado um desafio emergente para a comunidade cardiovascular internacional: tentar atrair o talento e o potencial da força de trabalho feminina para essa especialidade (principalmente para a cirurgia cardiovascular) ${ }^{18}$.

Uma pesquisa que comparou os impactos da feminização da medicina em quatro países de língua inglesa (Estados Unidos, Canadá, Reino Unido e Austrália) constatou que médicos e médicas não constroem a carreira da mesma maneira. Há persistentes diferenças de padrões e na escolha da especialidade entre os sexos que afetarão o futuro planejamento dos recursos de mão de obra médica ${ }^{13}$. Para as pesquisadoras Barbara Buddeberg-Fischer e Martina Stamm, apesar das grandes mudanças causadas pela feminização da medicina, as instituições de formação médica têm sido lentas em se adaptar à nova realidade. Nem as políticas institucionais, nem as condições de formação, nem os requisitos de seleção para as especialidades têm sido adaptados às necessidades femininas ${ }^{15}$.

Pensada como etapa exploratória de uma discussão sociológica mais ampla sobre o processo de formação das mulheres médicas, a pesquisa bibliográfica que deu origem a este artigo buscou fazer uma revisão de literatura sobre as questões contemporâneas que envolvem a educação das mulheres nas escolas de Medicina. O objetivo deste artigo é apresentar algumas das principais questões abordadas pela comunidade acadêmica com respeito a essa problemática e dar visibilidade às estratégias adotadas por algumas instituições de ensino como tentativa de promover a equidade de gênero no processo de formação.

\section{A PERSISTÊNCIA DA DISCRIMINAÇÃO DE GÊNERO}

Acesso e permanência já não são mais categorias de discriminação de gênero no curso de Medicina, mas o brilho da virada não deve esconder a fragilidade das conquistas e a persistência das desigualdades ${ }^{3,8}$. Embora as mulheres tenham conquistado importante espaço na medicina, esse deslocamento de fronteiras, como diz Souza-Lobo, "não eliminou a hierarquia de gênero"19(p.171). Sem dúvida, observam-se modificações importantes, mas "[...] é aqui que a construção de novas problemáticas tratando de articular a subordinação no trabalho e a hierarquia de gênero se faz necessária"19(p.159). Para descrever a nova tendência com relação à força de trabalho feminina, é preciso falar também das segregações escondidas que permeiam as instituições de ensino; e também da permanência de um conjunto de determinações sociais que atribuem ao trabalho do homem um peso e um valor maior do que ao trabalho da mulher ${ }^{19,20}$.

As mesmas pesquisas que apontam a crescente feminização da medicina indicam também a teimosa persistência da discriminação e do hiato de gênero na profissão. Pesquisas 
que se dedicam a estudar e comparar o processo de formação de homens e mulheres na medicina concluem que as mulheres médicas sofrem significativo impacto negativo provocado por estereótipos sexistas e discriminação de gênero já durante os anos de formação ${ }^{12,14,15,21}$. Formas sutis de discriminação encontram-se profundamente enraizadas na cultura médica e fazem com que homens e mulheres não estejam em situação de igualdade. A discriminação de gênero apresenta-se através de barreiras (diretas e indiretas) que impedem as mulheres de ascender na carreira em condições de igualdade com os homens e reproduzem espaços de formação demarcados por sexo ${ }^{22,23}$.

Essa segregação ocupacional iniciada durante o processo de formação reproduz guetos masculinos e femininos no interior da profissão e limita o acesso das mulheres a especialidades e áreas de maior prestígio e remuneração ${ }^{13,16,20,24}$. Dessa forma, a estrutura das distâncias entre o valorizado/desvalorizado na relação de dominação entre os sexos permanece, apesar das mudanças. Trata-se da "permanência dentro da mudança e pela mudança". Ao explicar esse fenômeno social, Bourdieu $^{24}$ esclarece que, quando uma profissão (ou determinada posição profissional) se feminiza, ocorre paralelamente uma deserção por parte dos homens. Isso porque, ao se tornar mais feminina, essa profissão passa também a ser menos valorizada, passa a ser vista como inferior. O pesquisador sustenta que, nesse caso, a desvalorização é duplicada, pois, além de a maior inserção feminina provocar a deserção em massa da força de trabalho masculina, acarreta também a desvalorização social ou econômica da profissão. Na medicina, o fenômeno da permanência dentro da mudança pode ser percebido através da evasão masculina de algumas especialidades que passaram a ser consideradas mais femininas. Sobre essa questão Bourdieu explica que:

As mudanças visíveis de condições ocultam, de fato, a permanência nas posições relativas: a igualização de oportunidades de acesso a índices de representação não deve mascarar as desigualdades que persistem na distribuição entre os diferentes níveis escolares e, simultaneamente, entre as carreiras possíveis. [...] Nas faculdades de Medicina, a porção de mulheres decresce à medida que se sobe na hierarquia das especialidades, algumas das quais, como a cirurgia, lhes estão praticamente interditadas, ao passo que outras, como a pediatria, ou a ginecologia, lhes estão quase que reservadas ${ }^{24}$ (p. 108 e 109).

No Brasil, a Pediatria e a Ginecologia foram as especialidades que mais sofreram evasão masculina ${ }^{8}$. Por outro lado, a presença feminina é muito limitada em áreas predominantemente masculinas, em especial nas cirúrgicas que utilizam tec- nologia avançada. São essas as áreas de maior prestígio, e precisamente nelas as mulheres estão em menor número $3,8,9,25,26,27$.

Ao tratar a questão das desigualdades de gênero que acompanham a formação escolar das mulheres, Duru-Bellat adverte que, embora alguns possam pensar que, ao menos nos países mais desenvolvidos, as questões referentes à discriminação de gênero parecem estar resolvidas, na verdade, não estão. Meninos e meninas, homens e mulheres continuam a ser orientados de forma diferente no contexto tanto doméstico como escolar ${ }^{28,29}$.

Baudelot e Establet ${ }^{20}$ explicam que a construção social da identidade pessoal (que começa na infância) não ocorre nas mesmas condições e nem da mesma forma para homens e mulheres. Há todo um direcionamento de expectativas sociais, denominadas estereótipos, que impregnam e internalizam no imaginário coletivo os papéis, representações e comportamentos esperados dos diferentes sujeitos sociais. Ao pesquisarem sobre o grande avanço feminino em todos os níveis de escolarização da sociedade francesa, Baudelot e Establet concluíram que o reconhecimento público da capacidade intelectual e profissional das mulheres não foi acompanhado pelo abandono dos estereótipos de gênero. Essas pesquisas revelaram que as representações e os comportamentos dos estudantes são ainda fortemente impregnados por estereótipos sexistas dominantes em todos os níveis escolares (inclusive no ensino superior).

Para Bourdieu, os efeitos duradouros e eficazes das manifestações de poder que dizem respeito à dominação masculina apresentam uma lógica paradoxal, pois, ao mesmo tempo em que se trata de uma relação imposta e extorquida, trata-se também de uma relação consentida, espontânea. Essa dualidade é fruto de um poder que se consolida não pelo uso da força ou violência física, mas, ao contrário, pela força simbólica de um trabalho lento, prolongado, silencioso e, quase sempre, invisível, que tem início precocemente na vida dos sujeitos masculinos e femininos e se perpetua por toda a existência de forma individual e coletiva, gerando o que o autor denomina "habitus sexuado"24.

As formas mais tradicionais de divisão sexual continuam a se reproduzir através dos modelos hierárquicos dos papéis atribuídos a homens e mulheres. A estrutura das organizações continua reforçando os estereótipos de gênero que valorizam o masculino em detrimento do feminino, mas a dinâmica de poder entre os gêneros pode ser cheia de dissimulações. Nem sempre as manifestações de poder se dão pela via da repressão. É preciso ter em conta toda a rede de "pedagogias" que, de forma aparentemente inocente e até democrática, constrói identidades, práticas e estereótipos. É preciso desconfiar dos gestos "tolerantes" e das concessões que mantêm determinados su- 
jeitos e práticas em lugares e funções socialmente demarcados como "naturais" a este ou àquele gênero ${ }^{30}$. $\mathrm{O}$ espaço a seguir apresenta algumas das práticas observadas nas escolas de Medicina que consolidam essa rede de "pedagogias" responsável pela persistência da discriminação de gênero na medicina.

\section{ESTEREÓTIPOS DE GÊNERO PRESENTES NAS PRÁTICAS DO COTIDIANO ESCOLAR}

Instituições de ensino que insistem em agir como há 50 anos, quando a medicina era dominada pelos homens, têm atuado como agentes promotores de barreiras para a progressão das carreiras femininas. A principal dessas barreiras tem sido a falta de acolhimento institucional para que as mulheres consigam combinar ao mesmo tempo um longo processo de formação e a construção da família. Isto porque as atuais políticas de formação forçam as mulheres a escolher entre a construção da carreira e a formação da família nos anos de residência $^{9,11,12,15,16,18,31}$. Resultados de pesquisas realizadas no Japão ${ }^{17}$, nos Estados Unidos ${ }^{18,34}$ e na Europa ${ }^{12,16,32,33}$ advertem que, a menos que ocorram mudanças político-estruturais no sentido de melhorar as condições de formação das mulheres médicas, o problema da escassez de especialistas médicos irá se agravar.

A estrutura do sistema de recrutamento, bem como do processo de seleção para a residência e para a medicina acadêmica pode atuar como instrumento de discriminação de gênero. A pesquisa realizada por Mason e $\mathrm{Ekman}^{31}$ dá algumas pistas sobre como essa discriminação ocorre. As pesquisadoras explicam que nas escolas médicas o processo de seleção para a residência ou para a pós-graduação com vistas à formação acadêmica é extremamente competitivo e exigente. Mulheres que fazem opção pela maternidade não são consideradas pelos professores e mentores como estudantes de alto potencial produtivo e provavelmente não passarão no exigente crivo do processo de seleção. Ainda que não seja um critério legitimamente válido, a maternidade (e não a paternidade) torna-se o primeiro fator de corte no processo de seleção. Apoiados em valores e hierarquias machistas, os processos de seleção reforçam os estereótipos de gênero e utilizam estratégias que prejudicam as mulheres. Buddeberg-Fischer e Stamm ${ }^{15}$ explicam que o perfil exigido para a aprovação em determinadas especialidades é extremamente sexista, refletindo uma rígida e conservadora estrutura de seleção. Algumas mulheres entrevistadas por Isobel Allen ${ }^{12}$ comentaram que as candidatas que afirmaram fazer ligações telefônicas para monitorar o que acontecia com os filhos em casa e aquelas que pretendiam reduzir seu horário de trabalho para passar mais tempo com os filhos foram desclassificadas do processo de seleção para a residência. Foram consideradas impróprias para o cargo preten- dido. Temendo não serem levadas a sério pelos examinadores ou por seus futuros orientadores e mentores, as mulheres adiam ao máximo a decisão pela maternidade. Uma mulher entrevistada por Mason e Ekman denuncia o preconceito de gênero: “O preço que estamos pedindo que as mulheres paguem para ascender na carreira é extremamente elevado. Elas estão sendo forçadas a escolher de uma forma que a maioria dos homens não é convidada a escolher"34 (p.94).

A maior parte das mulheres não pretende abrir mão da maternidade para se dedicar exclusivamente à carreira. Além disso, aspira por um equilíbrio entre trabalho e vida familiar. Antecipando os muitos obstáculos que terão de enfrentar ao conciliar filhos, vida familiar, estudos e trabalho, muitas mulheres escolhem especialidades nas quais esse equilíbrio aparentemente seja mais possível e se abstêm de carreiras cuja formação seja muito longa e exigente, como as diversas especialidades cirúrgicas $7,8,12-15,17,18,25,27,31,32$.

Um dos fatores responsáveis pela permanência dos estereótipos de gênero no processo de seleção para a residência é a pequena representatividade feminina no corpo docente das especialidades tidas como "masculinas". A escassa presença de mulheres acadêmicas nessas especialidades implica menores chances de as mulheres conseguirem um orientador ou mentor que as incentive ou que lhes conceda uma vaga na residência ou nos estágios. É por isso que a importância de modelos femininos como mentoras e orientadoras não pode ser subestimada. Os estudos mostram que a tutoria serve como um suporte para a carreira de sucesso. Conselheiros podem ajudar ou prejudicar as carreiras de um estudante, e as mulheres poderão estar em desvantagem se ficarem sob a orientação de um mentor que tenha preconceitos de gênero $^{11,12,14,15,16,18,21,23,25,27,31,32,35}$.

Mason e Ekman explicam por que mentores com mentalidade machista são um entrave para a carreira das mulheres médicas. A razão está ligada aos estereótipos de gênero fortemente enraizados na cultura médica do corpo docente que acabam sendo repassados para as alunas. Em algumas situações, as mulheres são aconselhadas de maneira tal, que muitas são levadas a pensar que nem todas as especialidades são apropriadas para uma mulher (especialmente para uma mulher que é ou pretende ser mãe). Durante os anos iniciais de formação, tais conselhos podem ser um fator determinante para o encaminhamento futuro de suas carreiras. Outra situação apontada pelas autoras diz respeito a mentores e orientadores de mentalidade machista que deixam de escolher estudantes mulheres para serem suas orientandas por acreditarem que mulheres que têm filhos não darão conta de uma formação de longo prazo ou não serão capazes de satisfazer as exigências do campo de pesquisa ${ }^{31}$. 
Outro estereótipo de gênero que permanece arraigado no imaginário social tem a ver com a desvalorização do trabalho feminino quando comparado ao masculino. O descrédito, a desconfiança de que a mulher não seja capaz de realizar um trabalho com a mesma competência e qualidade que o homem podem ser observados na citação a seguir:

Diante da desconfiança na capacidade feminina aparece a ideia [sic] de que as mulheres, para conquistar algo, em termos profissionais, precisam comprovar que são mais competentes do que os homens e isto exige delas uma dedicação muito maior (frise-se que nenhum homem mencionou, durante as entrevistas, este ponto). Em geral, elas precisam de maiores esforços pessoais e investimento na carreira ${ }^{25}$ (p.4).

Uma pesquisa de abordagem qualitativa realizada por Lempp e Seale com estudantes de uma escola de Medicina britânica constatou que, apesar de os estudantes afirmarem que não há diferenças de gênero nas experiências vivenciadas durante a formação médica, os estudantes de ambos os sexos revelaram a existência de estereótipos de gênero através de suas próprias posturas e falas. Por exemplo, a maioria dos estudantes entrevistados nessa pesquisa tem interiorizada a ideia de que a cirurgia é uma especialidade dominada por homens porque exige maior força e resistência física. Ao mesmo tempo, dizem também que há uma série de qualidades que as mulheres trouxeram para a medicina e que algumas especialidades respondem melhor a elas, como Pediatria, Ginecologia e Obstetrícia ${ }^{36}$.

Como observado até aqui, a força da interiorização dos estereótipos de gênero se encontra presente nos espaços escolares mais avançados (nas práticas tanto dos professores quanto dos alunos). Por ocasião das escolhas quanto à formação profissional, as expectativas ligadas a papéis de gênero levarão a maior parte das jovens mulheres a optar por carreiras consideradas socialmente mais apropriadas ao sexo feminino. Antecipando as prováveis dificuldades que encontrarão para conciliar família e trabalho, as mulheres tenderão a adaptar sua escolaridade e carreira profissional a seu papel na família. Em outras palavras, a maior parte das jovens mulheres acaba por se excluir dos cursos ou especialidades de maior prestígio ${ }^{37}$.

É nesse sentido que as novas formas de hierarquização de gênero no interior da medicina precisam ser entendidas a partir não somente dos estudos da divisão sexual do trabalho, mas também da permanência das desigualdades nos diferentes níveis do sistema de ensino. É preciso entender como os indivíduos constroem suas perspectivas quanto ao futuro profissional, como escolhem seus estudos superiores e, ao fa- zerem isso, transpõem para esse novo nível de ensino as desigualdades sociais incorporadas historicamente.

Neste ponto das considerações é importante abrir espaço para algumas questões sociológicas que envolvem os processos de escolha perpetrados pelos sujeitos quando por ocasião de sua formação profissional. Nogueira ${ }^{38}$ ressalta que, aparentemente, à primeira vista, o processo de tomada de decisão quanto à trajetória escolar a ser seguida parece se basear em preferências e interesses pessoais, mas, na verdade, essas escolhas sofrem influência direta das bases sociais de origem. Para escolher o rumo de sua formação profissional, os sujeitos passam por um complexo processo de autosseleção (muitas vezes, imperceptível a eles mesmos), que, por sua vez, está intensamente relacionado às diferentes características sociais de origem, como classe social, etnia ou sexo. Existe no processo de escolha a confluência de múltiplas influências. Algumas delas de caráter subjetivo, como as preferências e aspirações pessoais, as expectativas e projetos de vida; e outras de caráter objetivo, como o capital escolar acumulado ao longo da trajetória escolar, as características do mercado de trabalho, o peso do capital cultural, econômico e social da família de origem, os padrões culturais e expectativas sociais (inclusive de gênero) que fazem parte do contexto histórico-social em que o indivíduo está inserido. Complementando o pensamento, para Norbert Elias ${ }^{39}$, a maior ou menor liberdade de escolha do indivíduo e, também, o alcance de sua margem de decisão diante de diferentes possibilidades de escolha estão intrinsecamente relacionados à posição que esse indivíduo ocupa no interior de determinada rede social. Por sua vez, a posição ocupada por esse indivíduo na sociedade implica ainda uma série de condicionantes, como a variação estrutural da sociedade e o período histórico em questão.

Como apontado na pesquisa de Nogueira ${ }^{40}$, tratar sobre o processo de tomada de decisão dos sujeitos quanto à formação profissional é uma tarefa complexa. Ainda que os longos anos de socialização familiar e escolar exerçam forte influência sobre a construção dos projetos e perspectivas pessoais, é fundamental considerar que os indivíduos estão sob a influência de outras múltiplas (e, até mesmo, aparentemente contraditórias) disposições. Segundo Lahire ${ }^{41}$, as disposições são forjadas e consolidadas através das experiências passadas interiorizadas do mundo social, mas essas disposições são também passíveis de transformação. Os indivíduos são produto das experiências passadas que foram incorporadas e interiorizadas. No entanto, socializados por diferentes instituições, em diferentes períodos de sua existência, os indivíduos acabam por interiorizar uma grande variedade de disposições heterogêneas e contraditórias. Com o tempo, as disposições vão se construindo e se transformando, se tornando mais duráveis ou menos durá- 
veis. Tudo depende da intensidade, do tempo de socialização e do contexto onde a experiência de socialização se passa. Ao tratar da escola de Medicina como um dos mais longos e eficientes ritos de passagem em termos de formação profissional, Becker ${ }^{4}$ comenta que o que vai transformar os jovens calouros em médicos não é simplesmente a aprendizagem das ciências básicas para a prática da medicina. A ciência e a habilidade no diagnóstico e tratamento de doenças não fazem o médico. Após passar por um longo processo de instruções, cerimônias e provações, os jovens neófitos devem interiorizar uma espécie de cultura médica e aprender a desempenhar o papel de médico no "círculo encantado" da medicina.

\section{EM BUSCA DA EQUIDADE DE GÊNERO}

O objetivo de falar sobre as segregações escondidas é despertar os gestores, os docentes e as instituições de ensino para a necessidade de mudanças nas escolas de formação médica. Algumas das pesquisas que chamam a atenção para a permanência de políticas e práticas impregnadas de estereótipos de gênero trazem também exemplos de instituições onde as mudanças em busca da equidade de gênero têm acontecido. Dentre as instituições de ensino destacadas nas pesquisas de Mason e Ekman, e MacMurray, encontram-se Eastern Virginia Medical School, East Tennessee State University, Georgetown University, Harvard University, Yale University, University of California (Berkeley) University of Pennsylvania, University of Wisconsin ${ }^{13,31}$.

Dentre as mudanças políticas atualmente implantadas nessas universidades, estão o incentivo a um melhor clima no ambiente de formação para as mulheres em especialidades médicas tidas como "masculinas" e o incentivo à igualdade e diversidade de gênero no corpo docente das instituições. A preocupação com o recrutamento e permanência de docentes mulheres nos altos cargos da medicina acadêmica levou a Academy of Medical Sciences (na Inglaterra) a recomendar maior flexibilidade na carreira das mulheres através da concessão de bolsas de pesquisa destinadas especialmente a facilitar o regresso após a licença-maternidade. Trata-se de um fundo de financiamento que tem ajudado as mulheres a manter a produtividade em pesquisa mesmo durante os anos de criação dos filhos ${ }^{13,31}$

Uma estratégia de flexibilidade para a formação das mulheres tem sido o part-time married women's training scheme. Trata-se de um programa de residência flexível que permite às mulheres que têm filhos a possibilidade de estudo e estágio em tempo parcial. Esse programa, desenvolvido em algumas escolas de Medicina da Inglaterra, Austrália e Estados Unidos, foi introduzido com o objetivo de reduzir as taxas de abandono das mulheres médicas na residência ${ }^{13,31}$.
Diversas universidades têm criado programas de apoio e orientação educacional que promovem espaços de aconselhamento e orientação de carreira para as mulheres desde o início do processo de formação e, principalmente, na transição para a residência. A pesquisadora Barbara Buddeberg-Fischer explica que o planejamento estratégico de carreira em um estágio inicial do processo de formação é um fator crucial para a formação das mulheres médicas (especialmente para as estudantes que desejam ser mães) ${ }^{14}$.

Outra estratégia de flexibilidade tem sido a criação de políticas de família e de redes de apoio para que as mulheres que são mães consigam manter um equilíbrio entre o cuidado com os filhos e as responsabilidades exigidas pela residência médica. Creche em tempo integral em local próximo de onde as mães se encontram, sala de lactação para as mulheres residentes que amamentam e apartamentos dentro da universidade, reservados às estudantes de residência que têm filhos, são algumas das estratégias que têm feito parte dessas redes de apoio $^{31}$

Modificações na cultura médica do corpo docente e do corpo administrativo das instituições são a alavanca inicial para mudanças na estrutura política das faculdades de Medicina ${ }^{14,15}$. Uma das mudanças de maior impacto tem sido o incentivo a uma cultura médica que proporcione a equidade de gênero e, com respeito a essa questão, há indícios de que as atitudes estão mudando ${ }^{31}$. Se a equidade de gênero em termos de chances de carreira deve ser alcançada, medidas especiais de apoio terão de ser adotadas ${ }^{14}$.

\section{CONSIDERAÇÕES FINAIS}

Neste espaço de considerações finais me aproprio de um pensamento de Norbert Elias para dizer que entendo que, no que se refere às questões de gênero, a crescente inserção das mulheres na medicina "[...] é sinal de uma mudança comportamental de grandes proporções" ${ }^{\prime 42}$ (p.83). Ainda que seja o produto (não acabado) de transformações lentas e graduais, neste início do século XXI, a sociedade tem vivenciado a fronteira de uma longa curva no processo de transição da história da medicina. Nas últimas décadas, tem se rompido o hiato de gênero que, durante séculos, cerceou a entrada das mulheres na profissão médica. De uma profissão dominada pelos homens, a medicina passa a ser uma profissão onde a maioria dos novos médicos licenciados são mulheres.

Diante do fato de as mulheres estarem se tornando a principal força de trabalho na medicina, instituições e autoridades de formação serão compelidas a criar recursos e mecanismos de formação alternativos se quiserem solucionar o problema da escassez de profissionais médicos em determinadas espe- 
cialidades. Qualquer esforço para corrigir a má distribuição de profissionais por especialidade deve incluir as mulheres. Deve procurar descobrir quais são os fatores que influenciam as preferências na escolha da especialidade por parte das estudantes de Medicina e deve procurar entender por que as mulheres não têm sido atraídas para determinadas especialidades.

As instituições serão forçadas a promover mudanças a fim de atender às novas necessidades quanto ao perfil da profissão médica em termos de feminização e estilo de vida moderno. É tempo de reconsiderar alguns dos valores arraigados que reforçam a hierarquia da tradicional ordem das escolas de Medicina e dos programas de residência.

A pesquisa não contou com apoio de organizações de fomento.

\section{REFERÊNCIAS}

1. Cremesp. Levantamento mostra predomínio crescente de mulheres médicas. Jornal do Cremesp [on line]. 2010. 267 [capturado 16 jun. 2010]; 5. Disponível em: http:/ /www. cremesp.org.br $/$ ?siteAcao=Jornal\&id=1251

2. Cremesp. Mulheres médicas são maioria entre os mais jovens. Jornal do Cremesp [on line]. 2011. 286 [capturado 20 nov. 2011]; 8. Disponível em: http: / / www.cremesp.org.br / $?$ siteAcao $=$ CentroDados\&acao $=$ detalhes_capitulos\&cod_ capitulo $=1$

3. Cremesp. Demografia Médica no Brasil: dados gerais e descrições de desigualdades. São Paulo: Conselho Regional de Medicina do Estado de São Paulo e Conselho Federal de Medicina, 2011.

4. Becker HS, Geer B, Hughes EC, Strauss AL. Boys in White: student culture in medical school. 9. ed. New Brunswick and London: Transaction Publishers, 2005.

5. Dall'ava-Santucci J. Mulheres e Médicas: as pioneiras da Medicina. Rio de Janeiro: Ediouro, 2005.

6. Mott ML, Muniz MA, Alves OSF, Maestrini, K, Santos T. Médicos e médicas em São Paulo e os Livros de Registros do Serviço de Fiscalização do Exercício Profissional (18921932). Ciência e Saúde Coletiva 2008; 13(3):853 - 868.

7. Machado MH. A participação da mulher no setor da saúde no Brasil: 1970-1980. Cadernos de Saúde Pública 1986; 2(4):449-465.

8. Machado MH, coord. Os Médicos no Brasil: um Retrato da Realidade. Editora Fiocruz: Rio de Janeiro; 1997.

9. Franco T, Santos EG. Mulheres e cirurgiãs. Revista do Colégio Brasileiro de Cirurgiões 2010; 37(1):72-77.

10. Machado MCS. A feminização da medicina. Análise Social [online]. 2003. 38 (166) [capturado 20 nov. 2011]; 127-137.
Disponível em: <http:/ /analisesocial.ics.ul.pt/document os/1218737757C9xYD6fb1Nz74ES3.pdf>.

11. Poppas A, Cummings J, Dorbala S, Douglas P, Foster E, Limacher M. Survey Results: A decade of change in professional life in cardiology: a 2008 report of the women in cardiology council. Journal of the American College of Cardiology [online]. 2008. 52 (25) [capturado 20 abr. 2011]; 2215-2226. Disponível em: <http://content.onlinejacc. org/cgi/reprint/52/25/2215.pdf $>$.

12. Allen I. Women doctors and their careers: what now? British Medical Journal [online]. 2005. 331(7516) [capturado 20 abr. 2011]; 569-572. Disponível em: <http://www.bmj. com/content/331/7516/569.full.pdf $>$.

13. Macmurray JE, Angus G, Cohen M, Gavel P, Harding J, Horvath J, Paice E; Schmittdiel J, Grumbach K. Women in Medicine: A four-nation comparison. JAMWA [online]. 2002. 57 (4) [capturado 20 abr. 2011]; 185-190. Disponível em: <http:/ /www.amc.nl/upload/teksten/medische $\% 20$ psychologie/publications /2002/McMurrayJAmMedWomanAssn.2002.pdf $>$.

14. Buddeberg-Fischer B, Stamm M, Buddeberg C, Buer G, Hämmig O, Knecht M, Klaghofer R. The impact of gender and parenthood on physicians' careers: professional and personal situation seven years after graduation. BMC Health Services Research Zurich [online]. 2010. 10 (40) [capturado 18 abr. 2011]; 1-10. Disponível em: <http:/ /www. biomedcentral.com/content/pdf/1472-6963-10-40.pdf >.

15. Buddeberg-Fischer B, Stamm M. The medical profession and young physicians' lifestyles in flux: challenges for specialty training and health care delivery systems. Swiss Med Wkly [online]. 2010;140 (13134) [capturado 20 abr. 2011]; 1-11. Disponível em: <http:/ / www.buddeberg-praxis.ch/_pdf/bb/SMW_2010b.pdf>.

16. Soethout MBM, Ten Cate TJ, Van Der Wal G. Factors Associated with the Nature, Timing and Stability of the Specialty Career Choices of Recently Graduated Doctors in European Countries, a Literature Review . Medical Education [online] 2004; 9(24) [capturado 20 abr. 2011]; 1-9. Disponível em: <http://www.med-ed-online.org/res00114.htm>.

17. Fukuda Y, Harada T. Gender differences in specialty preference and mismatch with real needs in Japanese medical students. BMC Medical Education [online]. 2010;10(15) [capturado 06 ago. 2011]; 1-7. Disponível em: <http:/ / www. biomedcentral.com/content/pdf/1472-6920-10-15.pdf $>$.

18. De Maria AN. Cardiology Workforce Revisited. Journal of the American College of Cardiology [online]. 2008; 51(20) [capturado 06 ago. 2011]; 1986-1988. Disponível em: <http:/ / content.onlinejacc.org/cgi/reprint/51/20/1986.pdf>. 
19. Souza-Lobo E. A classe operária tem dois sexos. São Paulo: Brasiliense, 1991.

20. Baudelot C, Establet R. Allez les filles!. Paris: Seuil; 1992.

21. Taylor KS, Lambert TW, Goldacre MJ. Career progression and destinations, comparing men and women in the NHS: postal questionnaire surveys. British Medical Journal [online]. 2009; 338. [capturado 06 ago. 2011]; 1-12. Disponível em: <http://www.bmj.com/content/338/bmj.b1735.full.pdf

22. Hirata H, Kergoat D. A divisão sexual do trabalho revisitada. In: Maruani M, Hirata H (Orgs.). As novas fronteiras da desigualdade: homens e mulheres no mercado de trabalho. São Paulo: Senac, 2003. p. 111-123.

23. UIC. The University of Illinois at Chicago. Beyond parity workbook for action: transforming academic medicine through women's leadership. Center for Research on Women and Gender National Center of Excellence in Women's Health [on line]. Chicago, 2011, p.1-70. [capturado em 20 abr. 2011]. Disponível em: <http://www.uic. edu/orgs/womenshealth/bp_workbook156.pdf $>$.

24. Bourdieu P. A dominação masculina. 5. ed. Rio de Janeiro: Bertrand do Brasil; 2007.

25. Santos TS. Divisão sexual do trabalho na profissão médica e atividades Acadêmicas. VIII Congresso Iberoamericano de Ciência, tecnologia e gênero [on line]. abril de 2010. p. 4. [capturado 18 ago. 2011]. Disponível em: <http://200.134.25.85/eventos/cictg/conteudo_cd/E7_Divis\%C3\%A3o_Sexual_do_Trabalho_na_ Profiss \%C3\%A3o_M\%C3\%A9dica.pdf $>$.

26. Cruz EMTN. "Confesso que vivi": quatro décadas dedicadas ao estudante de Medicina. Cadernos ABEM 2010; 6: 55-61.

27. Siqueira VHF, Rocha GWF. A construção de diferenças de gênero entre estudantes de medicina. Cadernos Pagu 2008; 30:231-268

28. Duru-Bellat M. "Ce que La mixité fait aux élèves », Revue de l'OFCE [on line]. 2010; 3(114) [capturado 18 jun. 2012]; 197-212. Disponível em: http://www.cairn.info/resume. php?ID_ARTICLE=REOF_114_0197

29. Duru-Bellat M. "La (re)production des rapports sociaus de sexe: quelle place pour l'institition ecolaire ? », Travail, genre et sociétés [on line]. 2008; 1(19) [capturado 18 jun. 2012]; 131-149. Disponível em: http://formation.fsu.fr/IMG/ pdf_Duru-Bellat_La_re_production_des_rapports_sociaux_ de_sexe_-_quelle_place_pour_1_institution_scolaire.pdf

30. Louro GL. Gênero, sexualidade e educação. Petrópolis: Vozes; 2007.

31. Mason MA, Ekman EM. Mothers on the fast track: how a new generation can balance family and careers. New York: Oxford University Press; 2007.
32. Pöge K. Career goals and progressions of physicians during residency in Germany. 28th International Congress of the Medical Women's International Association [on line]. Münster, Germany. 2010 [capturado em 18 ago. 2011]. Disponível em: <http://www.mwia2010.net/postcongress / poege_5.10pm.pdf $>$.

33. Rusconi A, Solga H. Determinants of and obstacles to dual careers in Germany. Zeitschrift für Familienforschung [online]. 2007;19(3) [capturado 18 ago. 2011]; 311-336. Disponível em: <http://www.zeitschrift-fuer-familienforschung. de/pdf/2007-3-rusconi.pdf $>$.

34. Mason MA, Ekman EM. Mothers on the fast track: how a new generation can balance family and careers. New York: Oxford University Press; 2007. p.94.

35. Cruz EMTN. A escolha da especialidade em Medicina. Campinas; 1976. Doutorado [Tese] - Faculdade de Ciências Médicas, Universidade Estadual de Campinas.

36. Lempp H, Seale C. Medical students' perceptions in relation to ethnicity and gender: a qualitative study. BMC Medical Education [online]. 2006; 6(17) [capturado 18 ago. 2011]; 1-7. Disponível em: <http://www.biomedcentral. com/content/pdf/1472-6920-6-17.pdf $>$.

37. Duru-Bellat M. - L'école des filles. Quelle formation pour quels rôles sociaux? Paris, L'Harmattan, 1990.

38. Nogueira CMM. O processo de escolha dos estudos superiores: desafios para a investigação sociológica. II Colóquio Luso-brasileiro de Sociologia da Educação, Portalegre, Portugal, 2010.

39. Elias N. A sociedade dos indivíduos. Rio de Janeiro: Zahar; 1994.

40. Nogueira CMM. Dilemas na análise sociológica de um momento crucial das trajetórias escolares: o processo de escolha do curso superior. Belo Horizonte; 2004. Doutorado [Tese] - Universidade Federal de Minas Gerais.

41. Lahire B. Homem plural: os determinantes da ação. Petrópolis: Vozes; 2002.

42. Elias N. O processo civilizador: uma história dos costumes. Rio de Janeiro: Jorge Zahar; 1990.

\section{CONFLITO DE INTERESSES}

Declarou não haver.

\section{ENDEREÇO PARA CORRESPONDÊNCIA}

Rebeca Contrera Ávila

Caixa Postal 144 - Lavras

CEP 37200-000 - MG

E-mail: profrebecacontrera@gmail.com 